\title{
Refletindo sobre a convivência na UNIPAMPA campus Jaguarão: lazer, conhecimento e interação
}

\author{
Reflexión sobre la convivencia em UNIPAMPA campus Yaguarón: ocio, \\ conocimiento e interacción
}

\author{
Reflecting on the convivence of UNIPAMPA Jaguarão campus: leisure, \\ knowledge and interactión
}

Matheus Machado Rochel ${ }^{1}$

Eugenio Manoel Silva Prego ${ }^{2}$

Alessandra Buriol Farinha ${ }^{3}$

\begin{abstract}
Resumo
Vinculada ao projeto "PAMPATUR: Hospitalidade, Turismo e Organização de Viagens", esta pesquisa teve como objetivo identificar as atividades de lazer praticadas, e as de interesse de acadêmicos da Universidade Federal do Pampa (UNIPAMPA) - campus Jaguarão. Para isso, foi realizada uma pesquisa através de questionário respondido por 43 discentes da universidade. A amostra foi constituída por acadêmicos de ambos os sexos com idade entre 17 e 27 (ou mais) anos dos cursos de Turismo, Letras, Pedagogia, História e Produção Política e Cultural. A elaboração do instrumento de coleta dos dados teve como base as seis categorias de Marcelino (1996): físico, intelectual, artístico, manual, social e turístico. Foram feitos levantamento e análise do tempo livre dos acadêmicos, livre de obrigações profissionais, familiares, acadêmicas, sociais e religiosas. Os resultados indicaram que existe interesse na prática de lutas, entre as atividades intelectuais se destaca frequente o consumo de informação através da internet, assim como assistir filmes/séries e ouvir música, atividades relacionadas a culinária, ir a festas, namorar, sair com amigos, passear na cidade, porém com interesse em turismo náutico. Através desta pesquisa foi possível identificar melhor as segmentações de lazer de interesse dos estudantes, estes resultados contribuirão para a elaboração de propostas de lazer no campus, e então elaborar atividades que atendam aos seus perfis. As atividades de lazer podem promover a socialização, o intercâmbio de conhecimentos e impressões. Nesse contexto, além de contribuir para bem-estar dos discentes, as atividades de lazer no campus podem contribuir para a permanência dos discentes na universidade, através das relações humanizadas e experiências significativas em Jaguarão.
\end{abstract}

Palavras-Chave: Lazer; Interação; Unipampa; Jaguarão.

\section{Resumen}

Vinculado al proyecto "PAMPATUR: Hospitalidad, Turismo y Organización de Viajes", esta investigación tuvo como objetivo identificar las actividades de ocio practicadas, y las de interés de académicos de la Universidad Federal de Pampa (UNIPAMPA) - campus Jaguarão. Para ello, se realizó una encuesta a través de un cuestionario respondido por 43 alumnos de la universidad. La muestra fue constituida por académicos de ambos sexos con edad entre 17 y 27 (o más) años de los cursos de Turismo, Letras, Pedagogía, Historia y Producción Política y Cultural. La elaboración del instrumento de recolección de datos tuvo como base las seis categorías de Marcelino (1996): físico, intelectual, artístico, manual, social y turístico. Se realizó levantamiento y análisis del

\footnotetext{
${ }^{1} 1$ Graduando em Tecnologia em Gestão de Turismo na Universidade Federal do Pampa (UNIPAMPA).Monitor do Laboratório de Turismo (Labtur). rochel.matheus2@gmail.com.

${ }^{2}$ Graduando em Tecnologia em Gestão de Turismo na Universidade Federal do Pampa (UNIPAMPA). Monitor do Laboratório de Turismo (Labtur). eugenio.prego@gmail.com.

${ }^{3} 3$ Doutora em Memória Social e Patrimônio Cultural. Docente do Curso superior de Tecnologia em Gestão de Turismo da Universidade Federal do Pampa (UNIPAMPA). alessandrafarinha@unipampa.edu.br.
} 
tiempo libre de los académicos, libre de obligaciones profesionales, familiares, académicas, sociales y religiosas. Los resultados indicaron que existe interés en la práctica de luchas, entre las actividades intelectuales se destaca frecuentemente el consumo de información a través de internet, así como ver películas / series y escuchar música, actividades relacionadas con la cocina, ir a fiestas, salir, salir con amigos, pasear en la ciudad, también con interés en turismo náutico. A través de esta investigación fue posible identificar mejor las segmentaciones de ocio de interés de los estudiantes, estos resultados contribuirán para la elaboración de propuestas de ocio en el campus, y luego elaborar actividades que atiendan a sus perfiles. Las actividades de ocio pueden promover la socialización, el intercambio de conocimientos e impresiones. En este contexto, además de contribuir al bienestar de los discentes, las actividades de ocio en el campus pueden contribuir a la permanencia de los alumnos en la universidad, a través de las relaciones humanizadas y experiencias significativas en Jaguarão.

Palabras claves: Interacción; Yaguarón, Ocio, Unipampa.

\begin{abstract}
Related to the project "PAMPATUR: Hospitality, Tourism and Travel Organization", this research had as objective to identify the leisure activities practiced, and those of interest of academics of the Federal University of Pampa (UNIPAMPA) - campus Jaguarão. For this, a research was carried out through a questionnaire answered by 43 university students. The sample consisted of academics of both sexes with ages between 17 and 27 (or more) years of the courses of Tourism, Literature, Pedagogy, History and Political and Cultural Production. The elaboration of the instrument of data collection was based on the six categories of Marcelino (1996): physical, intellectual, artistic, manual, social and tourist. They were surveyed and analyzed the free time of the academics, free of professional, family, academic, social and religious obligations. The results indicated that there is interest in the practice of fights, among intellectual activities, the consumption of information through the internet is frequent, as well as watching movies / series and listening to music, cooking related activities, going to parties, dating, going out with friends, stroll in the city, but with interest in nautical tourism. Through this research it was possible to better identify the segments of leisure of interest of the students, these results will contribute to the elaboration of proposals of leisure in the campus, and then to elaborate activities that suit their profiles. Leisure activities can promote socialization, exchange of knowledge and impressions. In this context, besides contributing to the well-being of the students, the leisure activities on campus can contribute to the permanence of the students in the university, through humanized relations and significant experiences in Jaguarão.
\end{abstract}

Keywords: Interaction; Jaguarão; Leisure; Unipampa.

\title{
1. Introdução
}

Vinculada ao projeto de ensino denominado "PAMPATUR: Hospitalidade, Turismo e Organização de Viagens", coordenado pela docente Alessandra Buriol Farinha desde 2017 (em andamento), esta pesquisa teve como objetivo identificar as atividades de lazer praticadas, e as de interesse de acadêmicos da Universidade Federal do Pampa (UNIPAMPA)- campus Jaguarão. O referido projeto propõe, dentre outras ações, a mobilização dos discentes da universidade em atividades e experiências de interação entre discentes, docentes e comunidade acadêmica em geral, tais como organização e execução de walking tours, visitas técnicas, assessoria em eventos acadêmicos, dentre outras, oportunizando a integração entre os discentes, lazer, cultura, experiência de ensinoaprendizagem, atuação profissional dos discentes do turismo, dentre outros resultados satisfatórios. 
Justifica-se a elaboração desta pesquisa, pois, além de propor um uso educativo para o espaço acadêmico, a pesquisa pode indicar necessidades e possibilidades de melhorar o convívio, a qualidade de vida, as relações interpessoais no âmbito do campus. Entende-se que a pesquisa corrobora com o PDI (Plano de Desenvolvimento Institucional, 2013) da Universidade Federal do Pampa, que considera a interação como um pressuposto epistemológico da construção do conhecimento. O PDI (2013) também enfatiza sobre a importância de ações pedagógicas inovadoras, centradas na realidade dos contextos sociocultural, educacional, econômico e político da região onde a universidade está inserida.

Assim, a presente pesquisa foi desenvolvida para que haja um entendimento do perfil e das principais inclinações dos discentes da universidade no que tange ao lazer. Para isso, foi realizada uma pesquisa através de questionário respondido por 43 discentes da universidade. A amostra foi constituída por acadêmicos de ambos os sexos com idade entre 17 e 27(ou mais) anos dos cursos de Turismo, Letras, Pedagogia, História e Produção Política e Cultural. A elaboração do instrumento de coleta dos dados teve como base as seis categorias de Marcelino (1996): físico, intelectual, artístico, manual, social e turístico. Foram feitos levantamento e análise do tempo livre dos acadêmicos, livre de obrigações profissionais, familiares, acadêmicas, sociais e religiosas.

O presente trabalho constitui, de acordo com Triviños (1987), de uma pesquisa de caráter descritivo, visando determinar as opiniões do público envolvido, e de abordagem quantitativa (survey), segundo Fonseca (2002), para idealizar o percentual de alunos, de todos os cursos, em cada uma das categorias da pesquisa. Fizeram parte da amostra, até o dia 16/11/2018, 43 acadêmicos da UNIPAMPA de ambos os sexos, com idade entre 17 e 27(ou mais) anos, sendo 26 do curso de Turismo, 5 do curso de Pedagogia, 6 do curso de Produção Política e Cultural, 4 do curso de História e 2 do curso de Letras.

A ferramenta de coleta de dados a ser utilizada foi por meio de um formulário online através do Google Forms, este teve como base as seis categorias de Marcelino (1996): Atividades Físicas, Atividades Intelectuais, Atividades Artísticas, Atividades Manuais, Atividades Sociais e Atividades Turísticas. A análise dos dados foi feita a partir da própria ferramenta de pesquisa que realiza automaticamente a tabulação dos dados.

\section{Breves reflexões sobre o lazer no contexto acadêmico}


Por não haver um consenso sobre o significado de lazer, ocorre certa dificuldade em pesquisar sobre o tema. Para Marcellino (2002 apud Paim et al.2004), lazer é toda e qualquer ação realizada pelo ser humano fora de seu cotidiano, no qual consegue manter com suas obrigações; enquanto Dumazedier (1973) define lazer como:

[...] conjunto de ocupações às quais o indivíduo pode entregar-se de livre vontade, seja para repousar, seja para divertir-se, recrear-se e entreter-se, ou ainda para desenvolver sua informação ou formação desinteressada, sua participação social voluntária ou a sua livre capacidade criadora após livrar-se ou desembaraçar-se das obrigações profissionais, familiares e sociais (DUMAZEDIER, 1973, p. 34).

Marcellino (2006), afirma que: "Não é possível se entender o lazer isoladamente, sem relação com outras esferas da vida social. Ele influencia e é influenciado por outras áreas de atuação numa relação dinâmica" (MARCELLINO, 2006, p. 14-15), e comenta ainda que é feita a utilização, sem critério, da palavra lazer, onde deve-se atender à alguns valores ligados a atitude e tempo, e também descansar, realizar recreação, divertimento e desenvolvimento social e pessoal, advindos do lazer.

Segundo Elias (1985) “O trabalho, de acordo com a tradição, classifica-se a um nível superior, como um dever moral e um fim em si mesmo; o lazer classifica-se a um nível inferior, como uma forma de preguiça e indulgência" (ELIAS, 1985, p. 106), onde o lazer era relacionado como uma complementação do trabalho, já Melo (2003) nos diz que "o lazer, numa escala hierárquica de necessidades humanas, seria menos importante que a educação, a saúde e o saneamento" (p. 29), mas apesar disso, o lazer ainda é de grande importância para a vida de qualquer pessoa, principalmente daquelas que dedicam várias horas de seu dia para atividades intensivas (como trabalhar ou estudar), proporcionando momentos de relaxamento ou até mesmo descontração.

O lazer age também no alívio para as tensões do dia-a-dia, causadas pela rotina dessas pessoas, fazendo com que "o combate ao stress físico, mental e psicológico" se tornem "aliados da boa saúde. E as atividades de lazer são formas de divertimento, descanso ou desenvolvimento que podem trazer inúmeros benefícios, não só para sua saúde física, como para sua saúde mental e psicológica, que são tão importantes quanto à saúde física.” (BORGES, Raquel .2015, grifo do autor).

É uma criação proveniente do período entre o final do século XIX e XX, quando momentos de descontração e relaxamento puderam ser aliados ao trabalho. Bacal (2003) afirma que a formação cultural de cada um estabelece maneiras diferentes de reconhecer, programar, agir e dar valor ao mundo, ou seja, a visão do material e imaterial, a autora também elenca algumas atividades de lazer com motivação cultural, como: “diversão, interesses culturais; congressos científicos; fazer ou completar cursos; visitar feiras ou 
exposições; conhecer novos lugares; conhecer pessoas; buscar aventuras amorosas; conviver com a natureza." (BACAL, 2003, p. 102).

[...] o tempo se deixa medir, em termos de vivência individual, por critérios subjetivos, isto é, pela intensidade com que cada um de nós o sente. Nesse sentido, cada momento vale pela densidade das emoções, pela gradação da afetividade de que está carregado. [...] mais do que a quantidade, assume importância a qualidade dos instantes vividos (BACAL, 2003, p. 15).

A autora, na citação acima, se refere à qualidade do tempo no lazer, no contentamento, na satisfação humana pela qualidade dos momentos compartilhados com quem se aprecia, ou até sozinho. Essa é a fundamental importância do lazer: seu poder de reestabelecer a integridade, não apenas física, mas emocional das pessoas. Nesse mesmo ponto de vista, é imprescindível acrescentar nesta reflexão, os valores educacionais que o lazer pode proporcionar. No Brasil, as preocupações com lazer surgiram, segundo Pessoa (2007), na década de 1970, nas universidades, e a partir desse momento, o lazer deixa de ser visto como uma oportunidade de descanso, e passa a ser um direito social de todo brasileiro, além de ser visto também, como uma forma de consumo. Buzacarini e Corrêa (2015) destacam que:

\footnotetext{
Muitos destes indivíduos (jovens) exercem uma ocupação (trabalho) durante o dia e frequentam um curso do ensino superior no período noturno, perfazendo uma dupla jornada, reduzindo suas possibilidades de descanso, divertimento e desenvolvimento pessoal e social como possiblidade de lazer. Dessa maneira, os estudantes universitários, em geral, teriam somente os finais de semana, feriados e as férias para os estudos, descanso e tempo disponível para usufruírem do lazer. (BUZACARINI e CORRÊA, 2015, p. 17).
}

Por não terem tanto tempo disponível, esse ponto poderia causar certa limitação às atividades de lazer que possam vir a serem oferecidas a esses universitários (em sua grande maioria jovens). Sousa e Nahas (2011) afirmam que os universitários, de forma geral, não têm um comportamento saudável no que tange a atividades físicas e lazer, o que se soma a hábitos que podem ser classificados como prejudiciais à saúde, como o tabagismo, alcoolismo e uso de outras substâncias. Considera-se neste contexto a importância da saúde física e mental de acadêmicos, e se propõe a reflexão sobre atividades de lazer e de que forma elas podem influenciar na permanência de um ambiente saudável, com discentes culturalmente, fisicamente e socialmente integrados.

\section{Resultados da pesquisa: lazer para discentes da UNIPAMPA Jaguarão}

De acordo com os dados coletados através da pesquisa, foi possível então observar que, dos discentes que responderam à pesquisa, 20 (46,5\%) possuem idades que variam 
entre 19 e 22 anos, enquanto 15 (34,9\%) têm 18 anos ou menos; além disso, comprovou-se que $23(53,5 \%)$ dos entrevistados se identificaram pelo sexo masculino e $20(46,5 \%)$ pelo sexo feminino. Já em relação aos cursos envolvidos na pesquisa, notou-se grande participação do curso de Turismo na pesquisa, representando 60,5\% (26 discentes) do total de participantes, enquanto o curso de Letras teve baixa participação, contando apenas com a participação de 4,7\% (2 discentes) do total.

Partindo então para a análise dos dados coletados, referente a cada uma das categorias presentes no questionário, onde são apresentadas (por meio de gráficos com número de respostas em cada atividade) as atividades praticadas e atividades que tenham maior interesse por parte dos acadêmicos, dentre cada uma das categorias:

\subsection{Atividades praticadas pelos acadêmicos:}

A seguir serão apresentados os resultados da pesquisa referentes às atividades praticadas pelos acadêmicos da Universidade, separados de acordo com sua categoria, e com os principais dentre os mais praticados.

Atividades Físicas, sendo as mais praticadas: realizar caminhadas, fazer alongamentos, jogar futebol e realizar pedaladas, conforme o Gráfico 01:

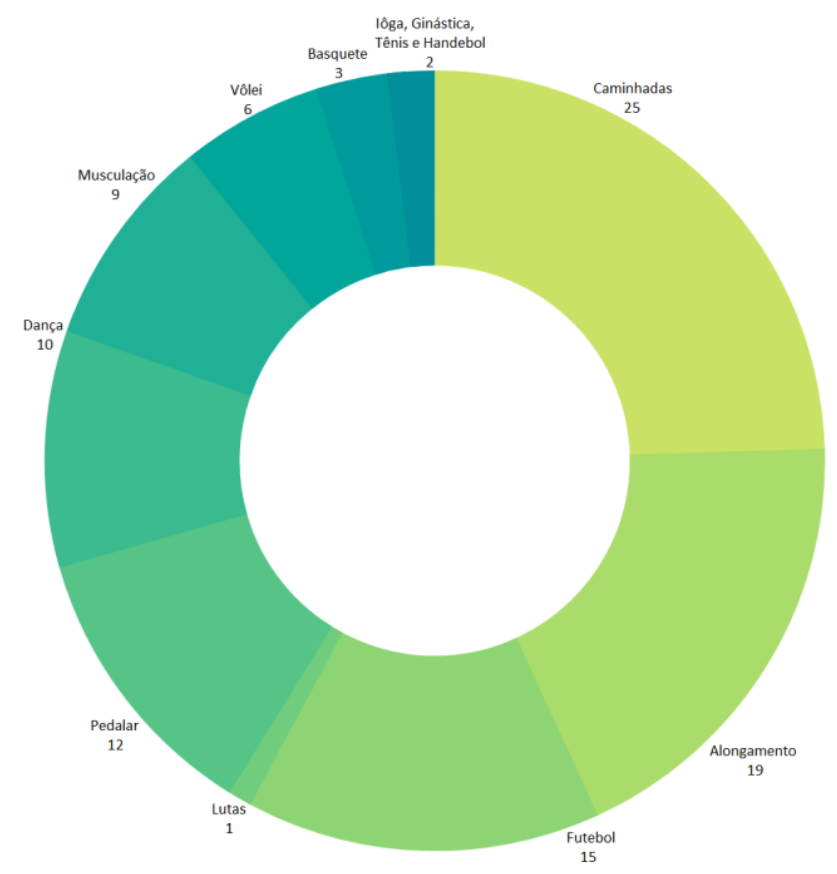

Gráfico 01: Distribuição de respostas referentes a atividades físicas que já são realizadas por discentes no campus. Fonte: Dos autores

Atividades Intelectuais, sendo as mais praticadas: uso da internet, prática de leituras e jogar videogame, conforme o Gráfico 02: 
RELACult - Revista Latino-Americana de Estudos em Cultura e Sociedade

Revista Latinoamericana de Estudios en Cultura y Sociedad | Latin American Journal of Studies in Culture and Society V. 05, ed. especial, abr., 2019, artigo no 1345| claec.org/relacult |e-ISSN: 2525-7870

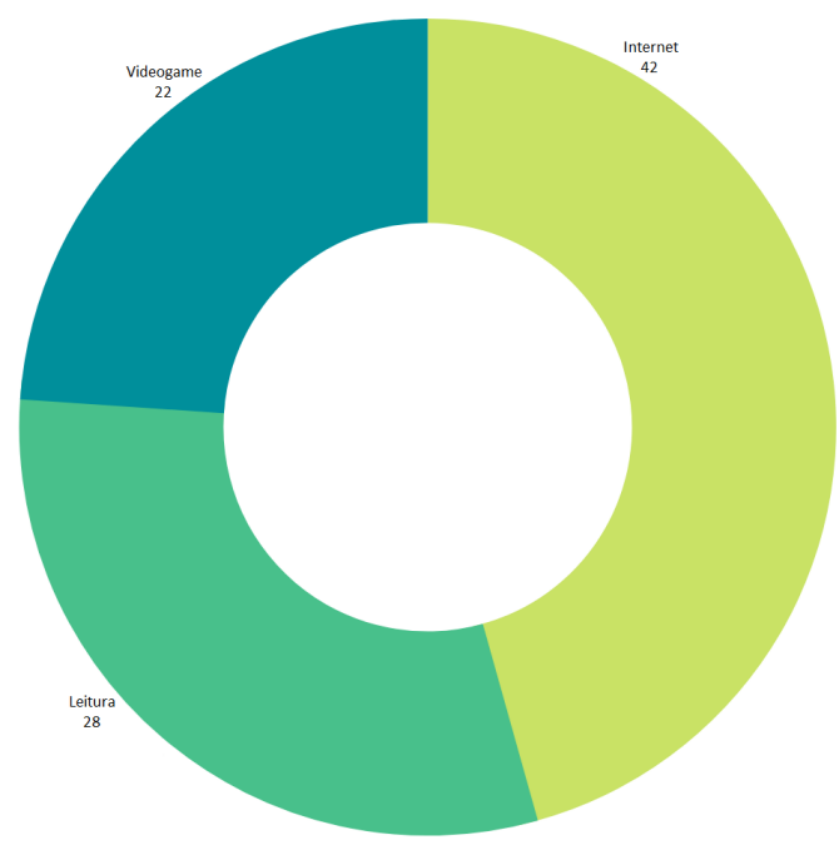

Gráfico 2 - Distribuição de respostas referentes a atividades intelectuais que já são realizadas por discentes no campus. Fonte: Dos autores

Atividades Artísticas, sendo as mais praticadas: ouvir música, assistir séries e/ou filmes e ir a shows musicais, conforme o Gráfico 03:

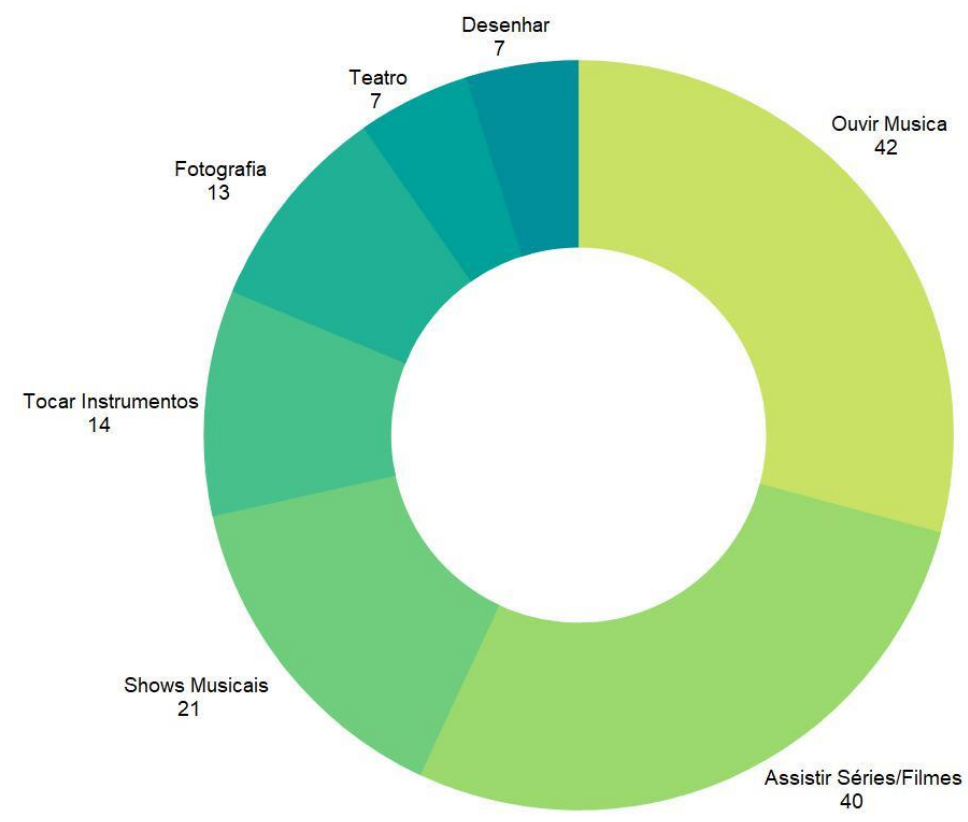

Gráfico 3 - Distribuição de respostas referentes a atividades artísticas que já são realizadas por discentes no campus. Fonte: Dos autores 
RELACult - Revista Latino-Americana de Estudos em Cultura e Sociedade

Revista Latinoamericana de Estudios en Cultura y Sociedad | Latin American Journal of Studies in Culture and Society V. 05, ed. especial, abr., 2019, artigo no 1345| claec.org/relacult |e-ISSN: 2525-7870

Atividades Manuais, sendo as mais praticadas: o cuidado de animais e a culinária, conforme o Gráfico 04:

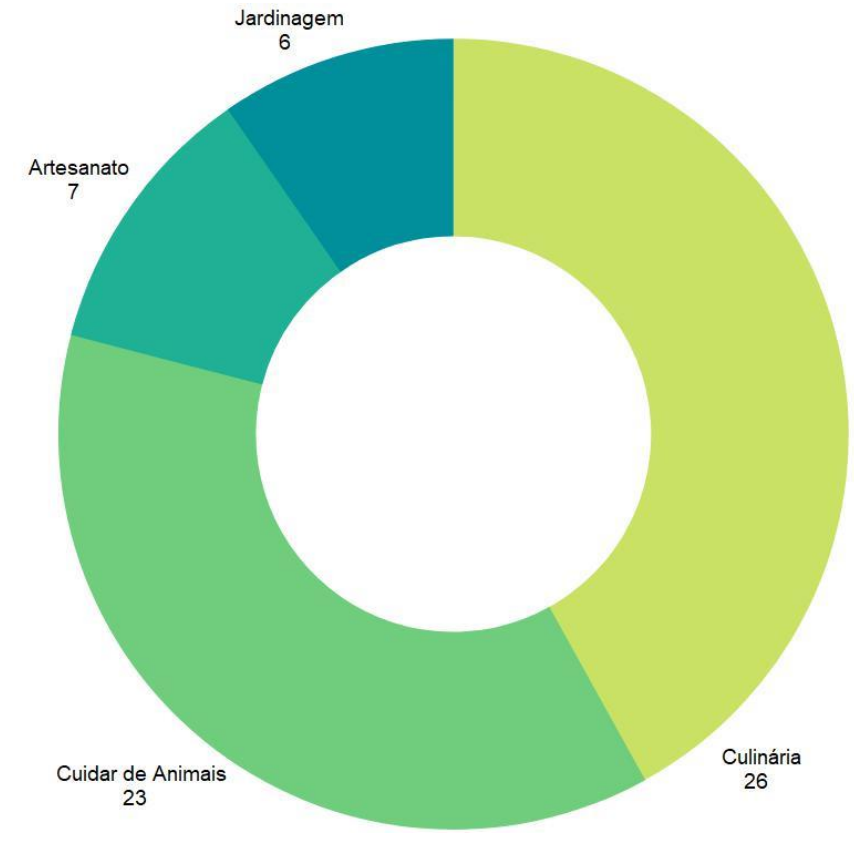

Gráfico 4 - Distribuição de respostas referentes a atividades manuais que já são realizadas por discentes no campus. Fonte: Dos autores

Atividades Sociais, sendo as mais praticadas: namorar e/ou ficar, sair com amigos e ir a festas, conforme o Gráfico 05:

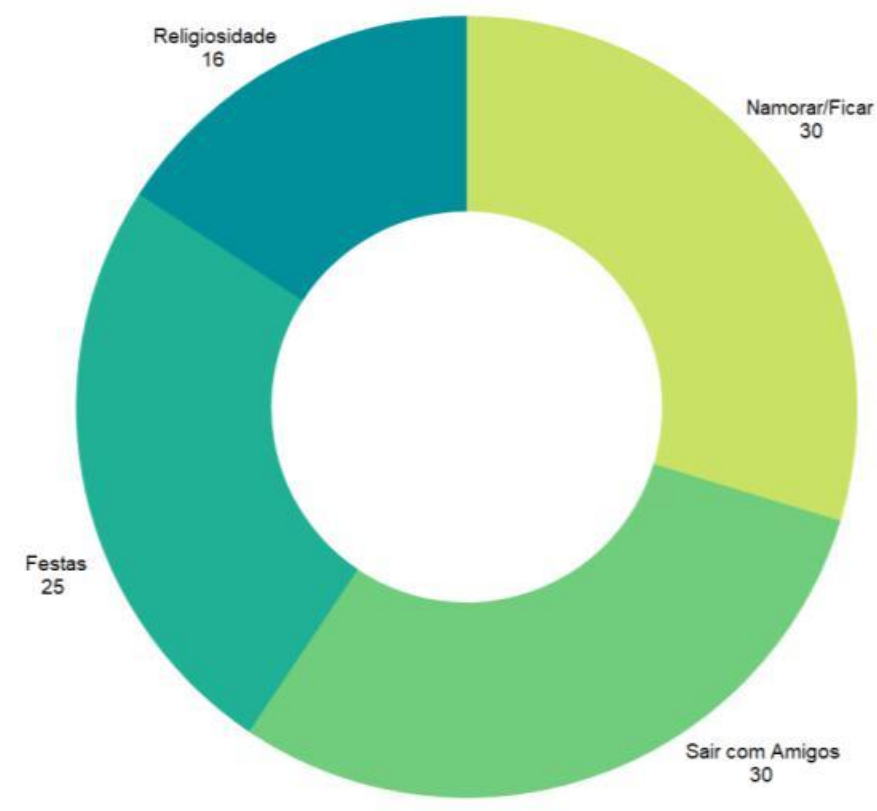

Gráfico 5 - Distribuição de respostas referentes a atividades sociais que já são realizadas por discentes no campus. Fonte: Dos autores 
Atividades Turísticas, sendo as mais praticadas: passeios na cidade, ir à praia e viagens na região, como demonstra o Gráfico 06.

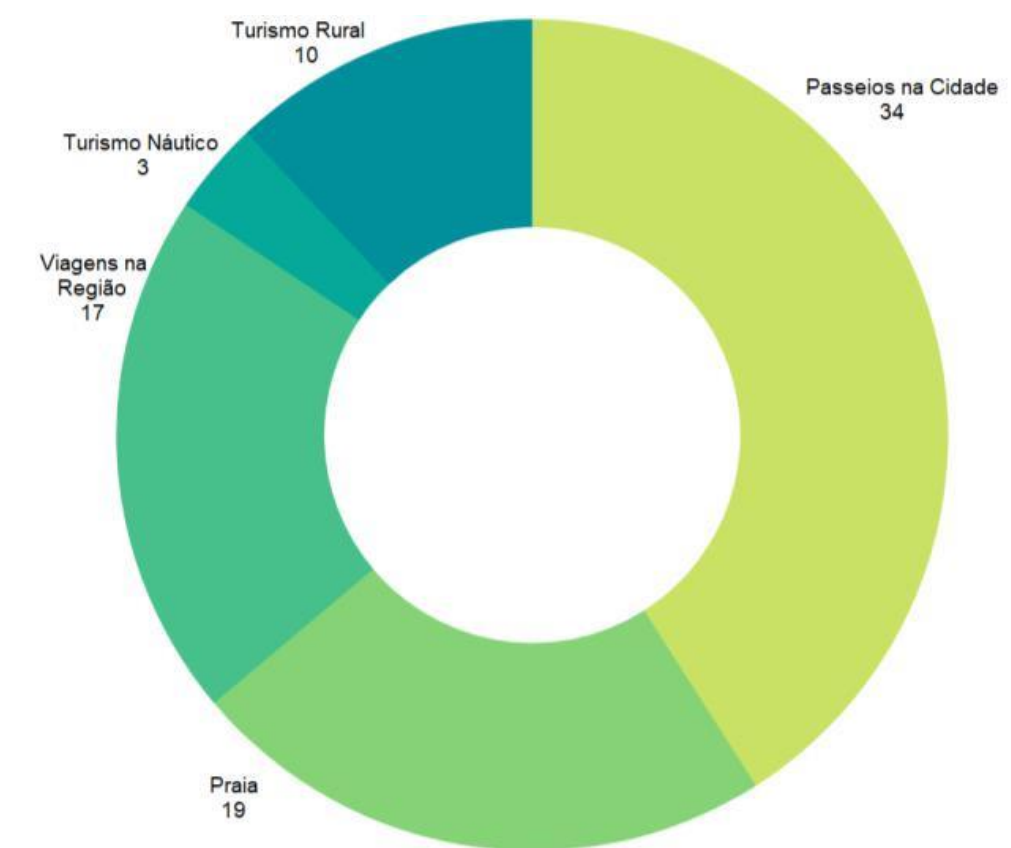

Gráfico 6 - Distribuição de respostas referentes a atividades turísticas que já são realizadas por discentes no campus. Fonte: Dos autores

A partir da análise das atividades de lazer já praticadas pelos acadêmicos, descritas acima, é possível afirmar que algumas delas podem ser incentivadas pela gestão do campus através de projetos e ações coletivas. Podem ser consideradas oportunidades de desenvolvimento: uma sala de projeções de cinema (já prevista pela gestão do campus, conforme a direção), cursos e minicursos de fotografia, utilizando a infraestrutura do campus, oficinas literárias, competições de jogos (xadrez, jogos de cartas, dentre outros), jardinagem no campus (hortaliças, árvores frutíferas e canteiros de flores), oficinas de artesanato utilizando matéria prima encontrada no campus (sementes, folhas, cascas de árvore), além de festas para acadêmicos.

\subsection{Atividades com maior interesse pelos acadêmicos:}

Por fim, serão apresentados os resultados referentes às atividades que contam com maior interesse pelos acadêmicos da Universidade, também separados de acordo com sua categoria e com os principais dentre os mais praticados. 
RELACult - Revista Latino-Americana de Estudos em Cultura e Sociedade

Revista Latinoamericana de Estudios en Cultura y Sociedad | Latin American Journal of Studies in Culture and Society V. 05, ed. especial, abr., 2019, artigo no 1345| claec.org/relacult | e-ISSN: 2525-7870

Atividades Físicas, sendo as com maior interesse: lutas, musculação, corridas e pedaladas, conforme o Gráfico 07:

Gráfico 7 - Distribuição de respostas referentes a atividades físicas as quais existe o interesse por parte dos

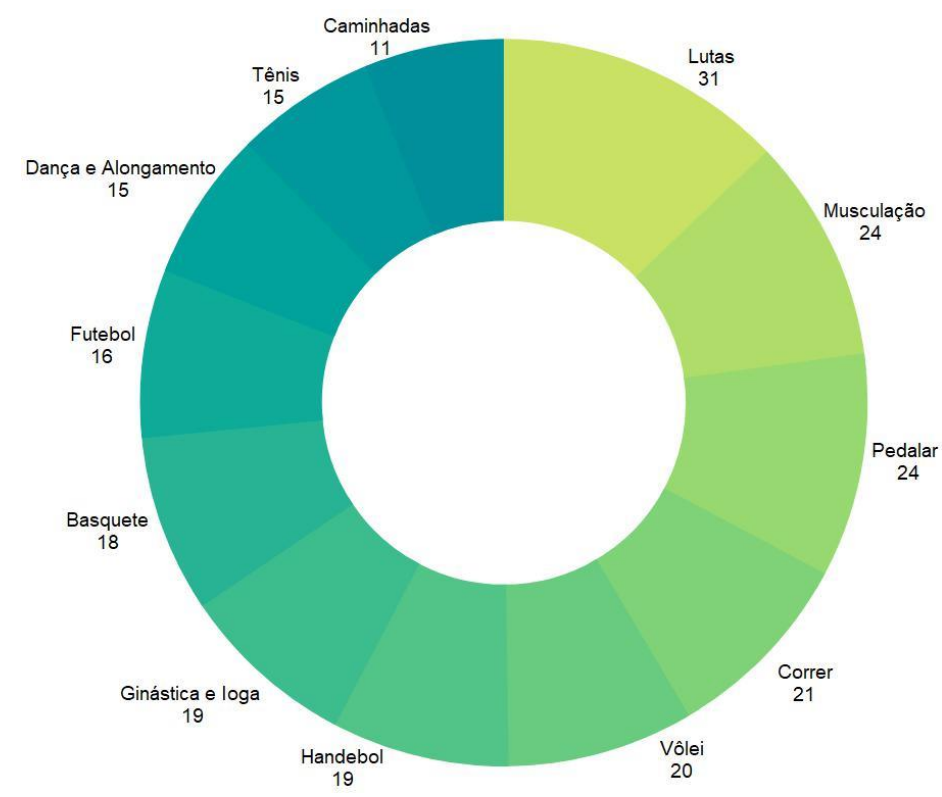

discentes no campus. Fonte: Dos autores

Atividades Intelectuais, sendo as com maior interesse: prática de leituras e jogar videogame, como demonstra o Gráfico 08:

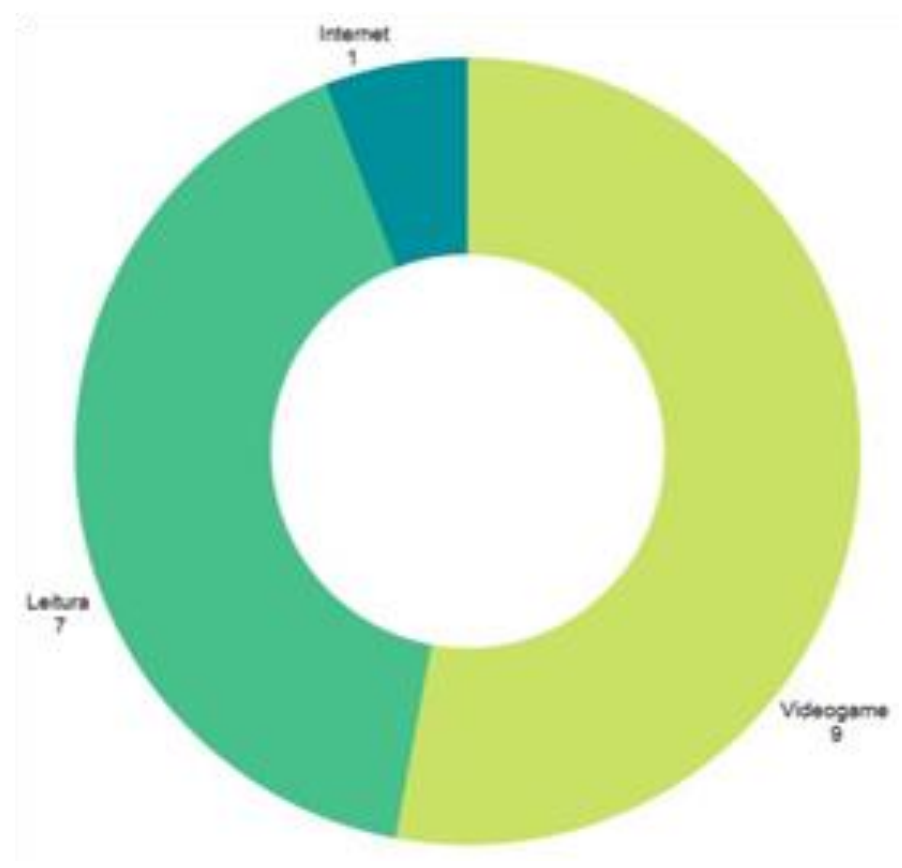

Gráfico 8 - Distribuição de respostas referentes a atividades intelectuais as quais existe o interesse por parte dos discentes no campus. Fonte: Dos autores 
RELACult - Revista Latino-Americana de Estudos em Cultura e Sociedade

Revista Latinoamericana de Estudios en Cultura y Sociedad | Latin American Journal of Studies in Culture and Society V. 05, ed. especial, abr., 2019, artigo no 1345| claec.org/relacult |e-ISSN: 2525-7870

Atividades Artísticas, sendo as com maior interesse: desenhar, tocar instrumentos, ir ao teatro e fazer fotografias, conforme o Gráfico 09:

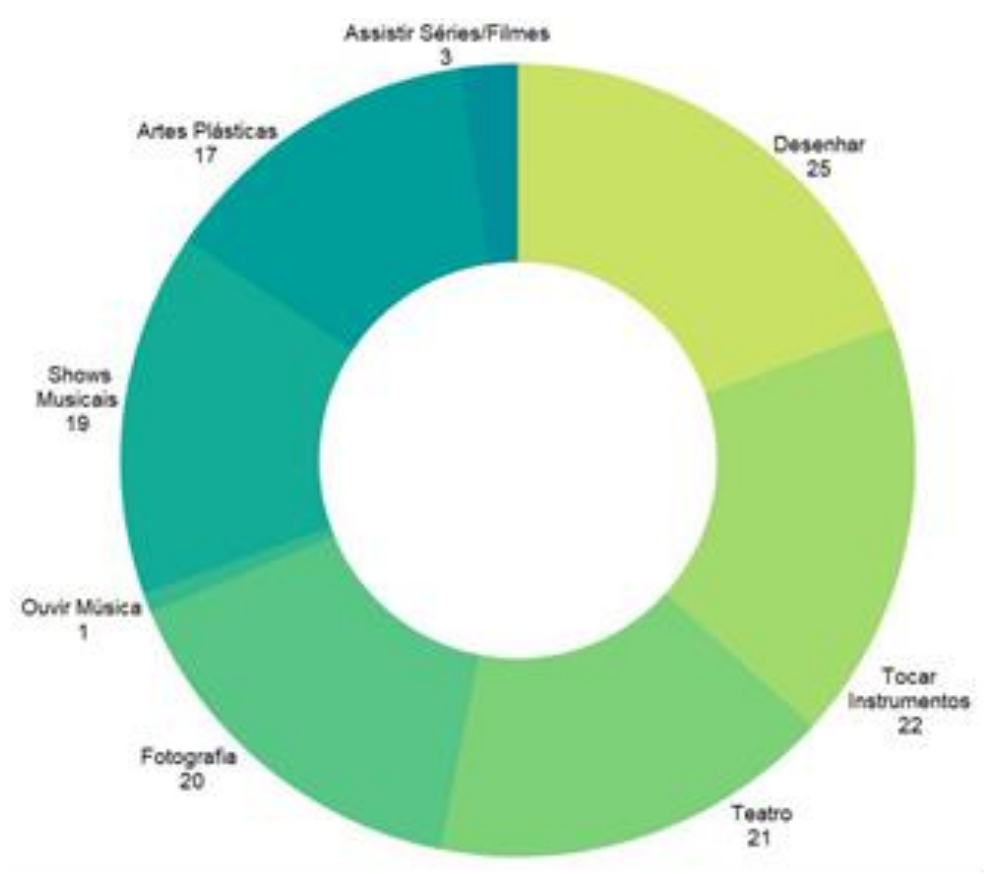

Gráfico 9 - Distribuição de respostas referentes a atividades artísticas as quais existe o interesse por parte dos discentes no campus. Fonte: Dos autores

Atividades Manuais, sendo as com maior interesse: cuidar de animais, artesanato e jardinagem, como mostra o Gráfico 10:

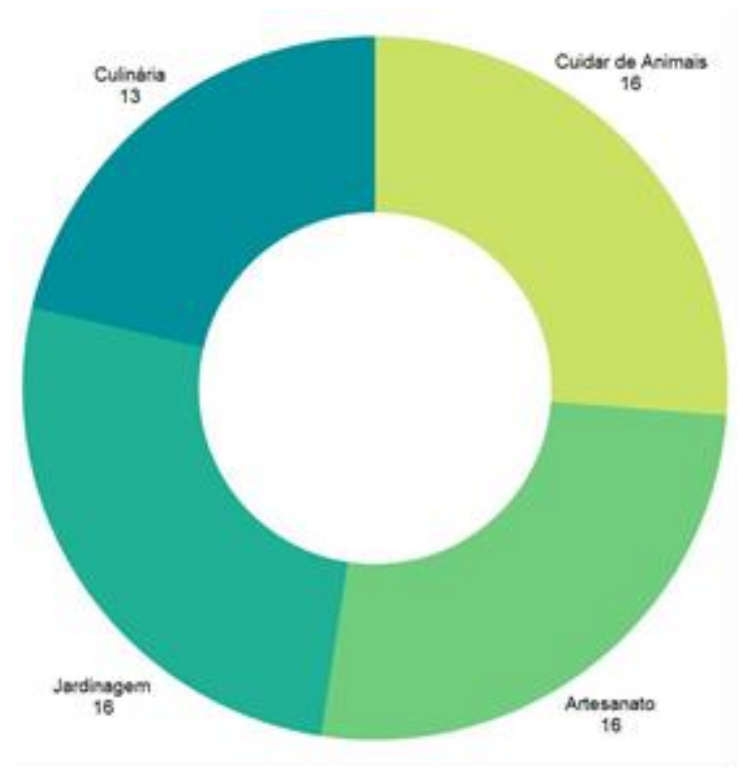

Gráfico 10 - Distribuição de respostas referentes a atividades manuais as quais existe o interesse por parte dos discentes no campus. Fonte: Dos autores 
RELACult - Revista Latino-Americana de Estudos em Cultura e Sociedade

Revista Latinoamericana de Estudios en Cultura y Sociedad | Latin American Journal of Studies in Culture and Society V. 05, ed. especial, abr., 2019, artigo no 1345| claec.org/relacult |e-ISSN: 2525-7870

Atividades Sociais, sendo as com maior interesse: ir a festas, sair com amigos e namorar e/ou ficar, conforme o Gráfico 11:

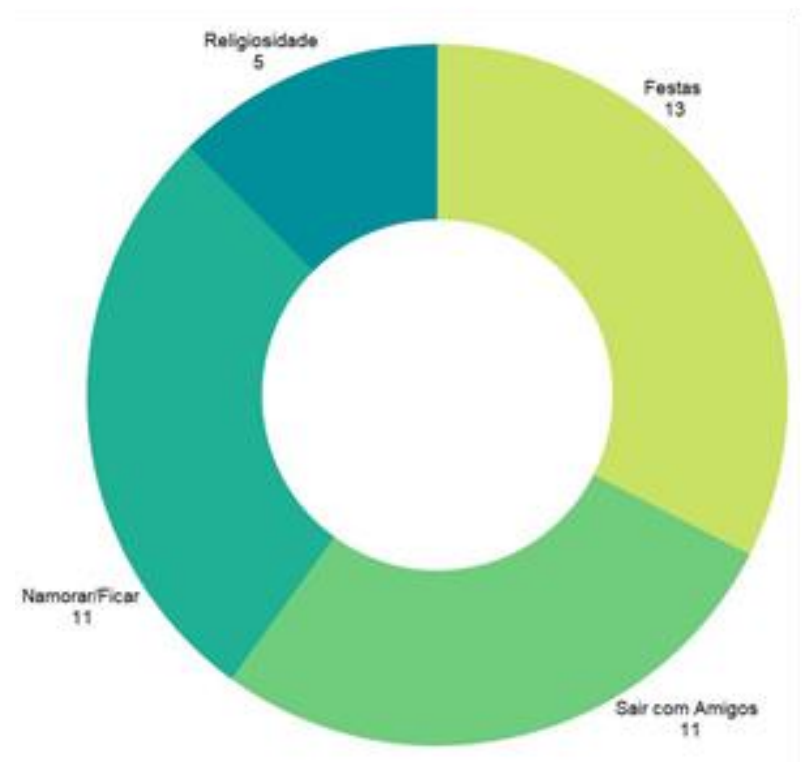

Gráfico 11 - Distribuição de respostas referentes a atividades sociais as quais existe o interesse por parte dos discentes no campus. Fonte: Dos autores

Atividades Turísticas, sendo as com mais interesse: turismo náutico, viagens na região, ir à praia e turismo rural, como demonstra o Gráfico 12.

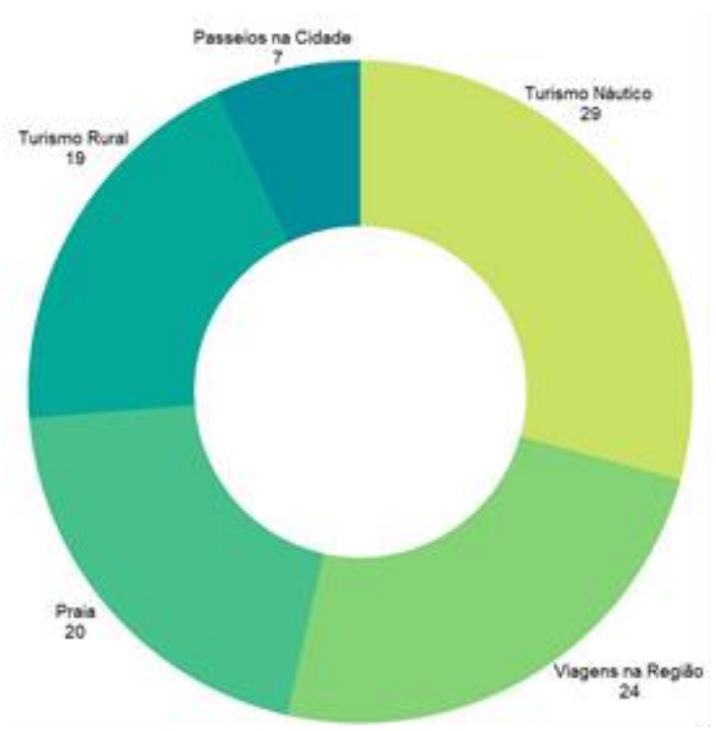

Gráfico 12 - Distribuição de respostas referentes a atividades turísticas as quais existe o interesse por parte dos discentes no campus. Fonte: Dos autores

Foi possível notar por meio desses dados que, ao mesmo tempo em que já há muita prática das mais diversas atividades, também há grande interesse por meio dos discentes, o que mostra a disposição dos mesmos para realiza-las. As expectativas dos discentes no que tange ao lazer são heterogêneas, e podem ser incentivadas. Os discentes podem, por exemplo, fazer oficinas para ensinar a outros discentes como tocar instrumentos, e isso pode se transformar em "festivais de talentos", culminando com apresentações no teatro - todos esses 
elementos despontam como interesses de lazer, conforme a pesquisa. A infraestrutura do campus pode ser mais bem aproveitada neste âmbito, por exemplo, com exposições de artesanatos produzidos pelos discentes na Galeria Intercultural Magliani ${ }^{4}$, ou utilizar o microônibus do campus para passeios de lazer na região.

Com o intuito de buscar respostas e reflexões acerca do lazer dos jovens acadêmicos, tendo como base os dados obtidos neste estudo, considera-se a possibilidade de criação atividades de lazer para atender os desejos dos jovens, que além de poderem proporcionar uma melhor qualidade de vida e disciplina a esses discentes, também poderiam, no caso das lutas e musculação, garantir autoconhecimento, defesa pessoal e condicionamento físico. $\mathrm{O}$ campus conta, em seu pátio interno, com amplo espaço aberto (Fotografia 1), que está apto para a prática de diversas atividades, e conta também com um profissional com formação em educação física, que poderia auxiliar na prática de atividades físicas.

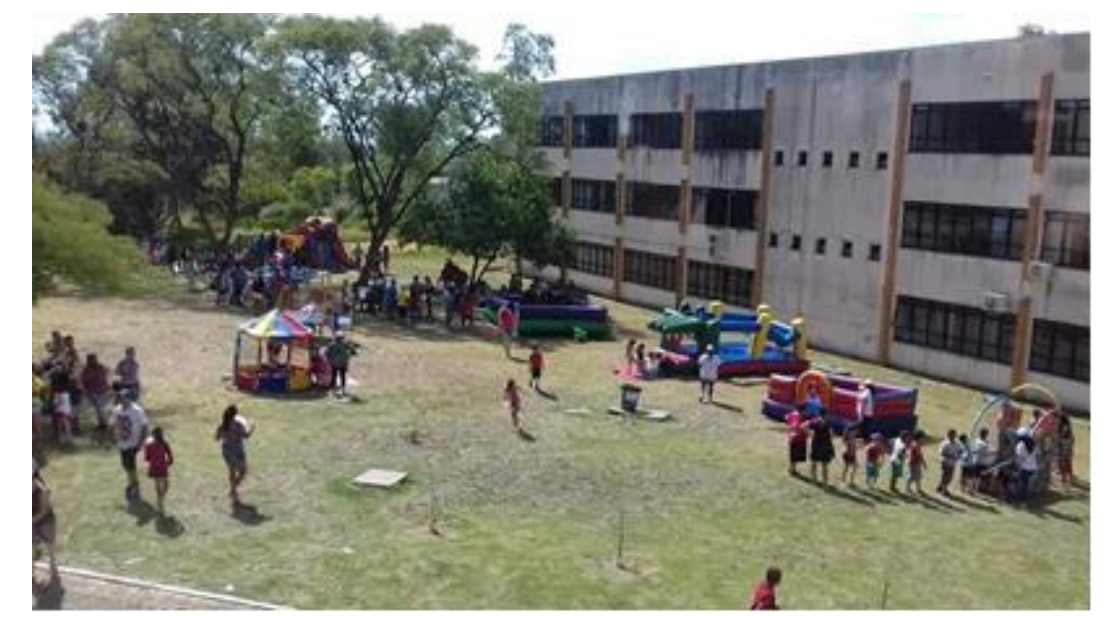

Inaugurada em novembro de 2017, encontra-se no subsolo do Campus Jaguarão. Fotografia 1 - Pátio Interno da UNIPAMPA Campus Jaguarão. Fonte: Página da Unipampa Jaguarão na rede social Facebook.

Recentemente foi criado no campus Jaguarão o Espaço de Convivência Dandara (Fotografia 2), inaugurado em novembro de 2018 por alunos do curso de Produção e Política Cultural através de um projeto coordenado pela docente Patrícia Schneider Severo. Este é um espaço para lazer no campus que conta com mesas onde podem ser realizados os trabalhos acadêmicos, rodas de conversa, jogos de mesa, e redes, que oferecem maior conforto para os frequentadores do local. 


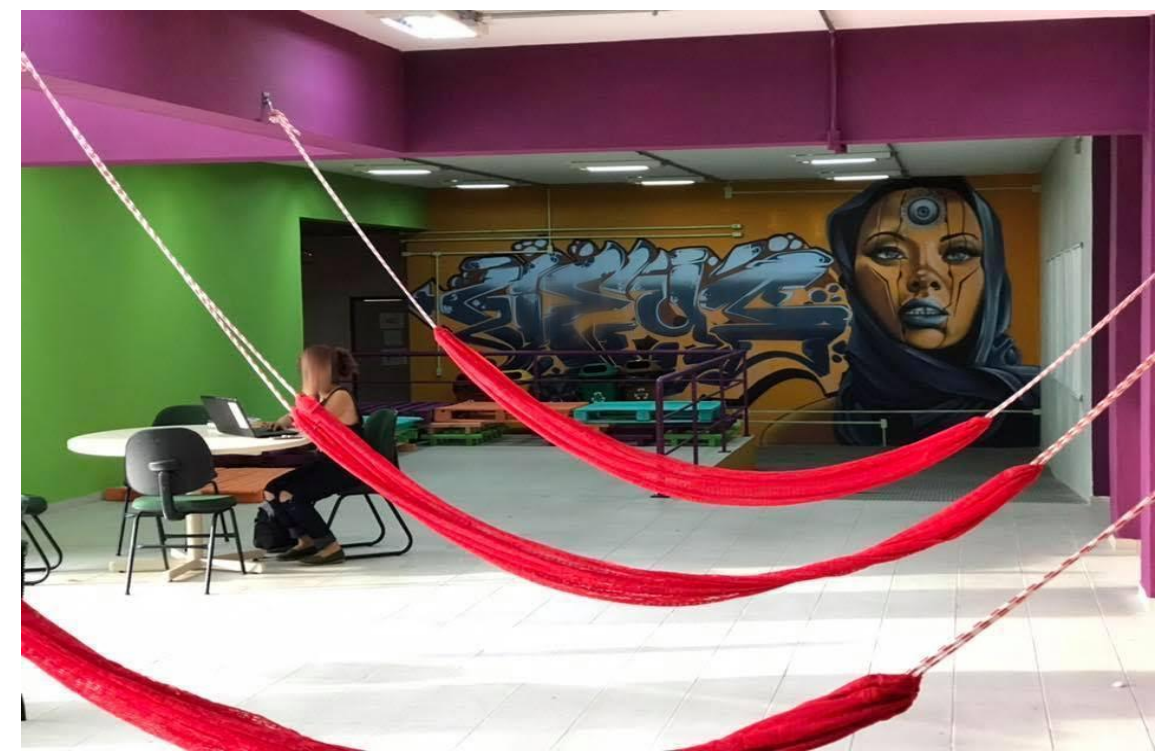

Fotografia 2 - Espaço de Convivência Dandara, UNIPAMPA Campus Jaguarão.

Fonte: Vinícius Rodrigues Aquino

\section{Considerações finais}

Espera-se que os resultados desta pesquisa, aliado a ações ligadas ao lazer no campus contribua para os processos de ensino-aprendizagem do curso de Tecnologia e Gestão de Turismo através da motivação, do incentivo à criatividade na organização das viagens, na possibilidade de atuação profissional na área, mesmo que em âmbito acadêmico, na vivência da teoria em experiências práticas de lazer. Entende-se que com propostas de lazer se pode promover uma maior integração intercursos, além de melhorias no relacionamento entre discentes, mais compreensão, solidariedade, altruísmo, propostas de socialização no campus que possam contribuir para a permanência dos discentes na UNIPAMPA campus Jaguarão.

Os resultados indicaram que existe interesse na prática de lutas, entre as atividades intelectuais se destaca frequente o consumo de informação através da internet, assim como assistir filmes/séries e ouvir música, atividades relacionadas à culinária, ir a festas, namorar, sair com amigos, passear na cidade, porém com interesse em turismo náutico. Através desta pesquisa foi possível identificar melhor as segmentações de lazer de interesse dos estudantes, estes resultados contribuirão para a elaboração de propostas de lazer no campus, e então elaborar atividades que atendam aos seus perfis. As atividades de lazer podem promover a socialização, o intercâmbio de conhecimentos e impressões. Nesse contexto, além de contribuir para bem-estar dos discentes, as atividades de lazer no 
campus podem contribuir para a permanência dos discentes na universidade, através das relações humanizadas e experiências significativas em Jaguarão.

\section{Referências}

BACAL, Sarah. Lazer e universo dos possíveis. 2 ed. São Paulo: Editora ALEPH, 2003.

BORGES, Raquel. A importância do lazer para nossa saúde e bem estar. Temporada Livre. Disponível em: <https://www.temporadalivre.com/blog/importancia-lazer-para-nossa-saudee-bem-estar/> Acesso em 12 jan. 2019.

BRANDÃO, Carlos. F. Atividades de tempo livre e atividades de lazer. $1^{\circ}$ Encontro da ALESDE - $\quad$ Curitiba, 2008.2 Disponível em: <http://www.alesde.ufpr.br/encontro/trabalhos/23.pdf/> Acesso em 10 jan. 2019.

BUZACARINI, Cíntia.; CORRÊA, Evandro. A. Lazer dos "estudantes universitários". Revista da Faculdade de Educação Física da UNICAMP, Campinas, v. 13, n. 2, p. 15-28,abr./jun. 2015.

DUMAZEDIER, Joffre. Lazer e cultura popular. São Paulo: Perspectiva, 1973.

ELIAS, Norbert \& DUNNING, Eric. A busca da excitação. Lisboa: Difel, 1985. Disponível https://drive.google.com/drive/folders/1nOdtzi_JaWAMvMjCIe6h2YxfZXn8IKfH

FONSECA, João. J. S. Metodologia da pesquisa científica. Fortaleza: UEC, 2002. Apostila. MARCELliNO, Nelson. C. Lazer e Educação. Campinas. São Paulo. Editora Papirus. 1987. 2006. $100 \mathrm{p}$. . Estudos do lazer: uma introdução / 4.ed. São Paulo. Autores Associados,

MELO, Victor. A. Introdução ao Lazer. Barueri SP: Manole, 2003.

PAIM, Maria. C. C. et al. Atividades de lazer praticadas por acadêmicos da UFSM no seu tempo livre. Revista Digital - Buenos Aires - Año 10 - N 69, 2004.

PESSOA, Amanda. R. R. Lazer, escola e juventude: algumas aproximações. Crato, 2007. 60 f. Monografia (Licenciado em Educação Física) - Universidade Regional Cariri. Disponível em: http://www.educadores.diaadia.pr.gov.br/arquivos/File/2010/artigos_teses/EDUCACAO_ FISICA/monografia/Lazer-escola-e-juventude.pdf/> Acesso em 9 jan. 2019.

PLANO DE DESENVOLVIMENTO INSTITUCIONAL (2014 - 2018) Universidade Federal do Pampa. Bagé, 2013.

SOUSA, Thiago e NAHAS, Markus Vinícius. Prevalência e fatores associados a menores níveis de prática de atividades físicas no lazer em estudantes de uma universidade pública do Estado da Bahia. Revista Brasileira de Atividade Física \& Saúde. V. 16. N. 02, 2011. Disponível em http://rbafs.org.br/RBAFS/article/view/627 Acesso em 14 de janeiro de 2018

TRIVIÑOS, Augusto. N. S. Introdução à pesquisa em ciências sociais: a pesquisa qualitativa em educação. São Paulo: Atlas, 1987. 